\title{
Association between Behavioural Psychological Symptoms of Dementia and Caregiver Burden in a South Indian Rural Community
}

\author{
Thasleem S.K. ${ }^{1}$, Sharma R. ${ }^{2}$, Shaji K.S. ${ }^{3}$ \\ ${ }^{1}$ Assistant Professor, Department of Mental Health, Nursing Government College of Nursing, Ernakulam, Kerala, \\ India, ${ }^{2}$ Professor, Department of Medicine, KS Hegde Medical Academy, Mangalore, Karnataka, India, ${ }^{3}$ Professor, \\ Department of Psychiatry, Government Medical College, Thrissur, Kerala, India
}

\begin{abstract}
Background: Behavioural and psychological symptoms of dementia (BPSD) and caregiver burden are two major issues in dementia patients. This study was conducted to estimate the association of BPSD with caregiver burden in patients with dementia living in a south Indian community.

Method: In a cross-sectional study done in Thalikkulam village, Kerala, 71 elderly of minimum 60 years and living with a primary caregiver for minimum one year were first selected with purposive sampling and screened with Brief Community Screening Instrument for Dementia. Then BPSD and caregiver burden were measured by Neuropsychiatric Inventory Questionnaire and Zarit Burden Interview respectively.

Result: Statistical analysis revealed that all patients (100\%) experienced at least one BPSD and apathy (76.1\%), delusion (74.6\%) and agitation (70.4\%) were the most common BPSD reported. Majority (93\%) of caregivers reported caregiver burden. Strong positive correlation $(p>0.05)$ was found between BPSD scores and caregiver burden scores.

Conclusion: The study found that BPSD and caregiver burden is common among community dwelling patients with dementia and have significant association with various socio demographic variables. This study recommends that while identifying dementia in community, health professionals need to focus on management of BPSD and caregiver burden which can influence the outcome of dementia.
\end{abstract}

Keywords: Behavioural and psychological symptoms of dementia (BPSD); Caregiver burden; rural community; Elderly; South India.

\section{Introduction}

The proportion of older persons to global population will increase from $8.5 \%$ today to more than $21 \%$ in $2050 .{ }^{1}$ Approximately $20 \%$ of elderly suffer from a mental disorder and dementia is the second commonest of these. ${ }^{2}$ Dementia is an overall term that describes a

\footnotetext{
Corresponding Author: Thasleem Sabith K.

Address: Assistant Professor, Government College of Nursing, Ernakulum, Kerala, India

Mobile Number: 9048550440

e-mail: thasleem.sabith@gmail.com
}

group of symptoms associated with a decline in memory or other thinking skills severe enough to reduce a person's ability to perform everyday activities. Worldwide, around 50 million people have dementia ${ }^{3}$ and every year, there are nearly 10 million new cases, equivalent to one new case of dementia in every 3.2 seconds are being diagnosed. ${ }^{4}$ It was the fifth leading cause of global death in $2016 .{ }^{5}$ Indian elderly population is currently the second largest in the world and there were 3.7 million elderly in India with dementia in 2010 and projected to rise to 7.6 million by $2030 .{ }^{6}$ South Indian states have the highest proportion of elderly in the country with Kerala at the peak with $12.6 \% .^{7}$ Thus south Indian states will be going to witness a significant rise in dementia cases in the coming years. 
Behavioural and psychological symptoms of dementia (BPSD) are heterogeneous range of psychological reactions, psychiatric symptoms and behaviours and are found in $90 \%$ patients with dementia. BPSD are distressing and difficult to manage, and have been associated with a poorer prognosis, faster illness progression, greater impairment in activities of daily living, increased hospitalization, and loss of quality of life for patients and their families. ${ }^{8}$

Caregiver burden is defined as a multidimensional response to the negative appraisal and perceived stress resulting from taking care of an ill individual. It threatens the physical, psychological, emotional and functional health and increases morbidity and mortality of the caregiver. More than $80 \%$ of dementia caregivers frequently reported high levels of stress and half of them were suffering from depression and anxiety.(Cooper $\mathrm{C}$ et al. 2014) $)^{9}$ Not much recent studies were found on the BPSD and caregiver burden among dementia patients in south India. This study was conducted to find the association between BPSD and caregiver burden among patients living in a south Indian rural community.

\section{Materials and Method}

Anon-experimental descriptivecross sectional design was adopted in this study. Elderly individuals living in Thalikkulam village of Thrissur district were screened with CSID BRIEF. Sample size was calculated using the formula and those who scored six or less in CSID BRIEF was selected by purposive sampling method. Seventy one elderly of more than 60 years of age and are living with a primary caregiver for a minimum of last one year were included in the study. Elderly who were diagnosed with vascular dementia or severe physical or psychiatric illnesses were excluded from the study.

Data collection tools: Brief Community Screening Instrument for Dementia (CSID-BRIEF):It has 13 self report items with two subscalesand developed by Hall et $\mathrm{al}^{10}$. Cognitive subscale has seven questions which checks recall, comprehension and orientation of patients. Informant subscale has six questions for caregivers. On the basis of total scores elderly were categorized to no dementia, probable dementia and dementia. Elderly belonged to dementia and probable dementia was selected for the study. The tool has good diagnostic accuracy, sensitivity, specificity, content validity (CVI0.90-0.95) and test retest reliability ( $\mathrm{r}-0.92)$.(Prince $\mathrm{M}$ et al. 2011). ${ }^{11}$

Socio demographic variables were assessed by a 16 item self-report tool developed by the researchers. The tool reported a good content validity (CVI 0.75-0.85).

Neuropsychiatric Inventory Questionnaire (NPIQ):It is an informant interview based instrument by JL Cummings. ${ }^{12}$ It measured the presence, severity as well as informant distress of twelve BPSD sub domains. NPI-Q reported good sensitivity, specificity, internal consistency, ${ }^{13}$ content validity (CVI 0.95- 0.98) and inter-rater reliability $(\mathrm{r}-0.87)$.

Zarit Burden Interview: It is a 22 item scale by Zarit et $\mathrm{al}^{14}$ and total score ranges from 0 to 88 and categorized to little or no burden, mild to moderate burden, moderate to severe burden and severe burden. The scale has good internal consistency, intra-class correlation, (Seng BK et al. 2010) $)^{15}$ content validity(CVI $0.85-0.95)$ and test-retest reliability ( $\mathrm{r}-0.88)$.

Findings: While doing descriptive analysis, it was found that most $(75 \%)$ of the patients were females with a mean age of $80.03+7.67$ years and $64.8 \%$ of them were educated up to primary school. Mean duration of dementia was 38.04 months. The mean age of the caregivers was $50.38+13.21$ years and most $(98.6 \%)$ of them were femalesand $36.6 \%$ of them were educated up to primary school.

BPSD and caregiver burden: Fig 1 shows the distribution of BPSD severity and distress among the patients reveals that apathy (76.1\%), delusion (74.6\%) and agitation $(70.4 \%)$ were the most common and severe BPSD found among the patients. At the same time, apathy $(74.6 \%)$, and delusion $(70.4 \%)$ were the most common distress producing symptoms. As shown in fig $2,93 \%$ caregivers reported some form of burden and out of that, $14 \%$ experience severe burden.

While analysing association, we found a strong positive correlation between BPSD severity and BPSD distress (Spearman's correlation, $\mathrm{p}<0.05$ ) among all sub domains. We also found that caregiver burden was positively correlated with BPSD total, delusion, hallucination, agitation, disinhibition, irritability, motor disturbances and night time disturbances. (Table 1). 
BPSD, caregiver burden and socio personal variables:

Table 1 describes the association between BPSD and socio personal variables shows that age was positively correlated with hallucination and nighttime behaviour, duration of dementia was correlated with delusion, hallucination, motor disturbances and night time disturbances (Spearman's correlation, $\mathrm{p}<0.05$ ). Analysis also revealed that hallucination and motor disturbances were significantly associated with patient's gender and hallucination, euphoria and motor disturbances was associated with patient's marital status (Mann Whitney $U, p<0.05)$. When analysed with Kruskal
Wallis $\mathrm{H}$ test, we found that agitation and disinhibition was significantly associated with type of family, hallucination and agitation were significantly associated with type of caregiver and delusion and irritability were significantly associated with type of accommodation of patients $(\mathrm{p}<0.05)$. (Table 2$)$.

Caregiver burden was found to be positively correlated with duration of dementia and with delusion, hallucination, agitation, depression, apathy, disinhibition, irritability, motor disturbances and night time disturbances (Spearman's correlation, $\mathrm{p}<0.05$ ). Care giver burden was found to be different in different levels of dependence (Kruskal Wallis $\mathrm{H}$ test, $\mathrm{p}<0.05$ ).

Table 1: Association between BPSD severity and distress, caregiver burden and socio personal variables

\begin{tabular}{|l|c|c|c|c|c|c|c|c|}
\hline \multirow{2}{*}{ BPSD Severity } & \multicolumn{2}{|c|}{ BPSD Distress } & \multicolumn{2}{|c|}{ Caregiver burden } & \multicolumn{3}{|c|}{ Age } & \multicolumn{2}{c|}{ Duration of dementia } \\
\cline { 2 - 9 } & $\mathbf{r}$ & $\mathbf{p}$ & $\mathbf{r}$ & $\mathbf{p}$ & $\mathbf{r}$ & $\mathbf{p}$ & $\mathbf{r}$ & $\mathbf{p}$ \\
\hline BPSD total & $0.96^{*}$ & $<0.0001$ & $0.58^{*}$ & $<0.0001$ & 0.07 & 0.54 & 0.23 & 0.06 \\
\hline Delusion severity & $0.88^{*}$ & $<0.0001$ & $0.42^{*}$ & $<0.0001$ & -0.10 & 0.40 & $0.23^{*}$ & 0.05 \\
\hline Hallucination severity & $0.98^{*}$ & $<0.0001$ & $0.36^{*}$ & 0.002 & $0.26^{*}$ & 0.03 & $0.28^{*}$ & 0.02 \\
\hline Agitation severity & $0.91^{*}$ & $<0.0001$ & $0.39^{*}$ & 0.001 & -0.11 & 0.38 & -0.06 & 0.65 \\
\hline Depression severity & $0.92^{*}$ & $<0.0001$ & 0.23 & 0.06 & -0.09 & 0.48 & -0.03 & 0.84 \\
\hline Anxiety severity & $0.96^{*}$ & $<0.0001$ & 0.14 & 0.24 & -0.03 & 0.83 & -0.21 & 0.09 \\
\hline Euphoria severity & $0.97^{*}$ & $<0.0001$ & 0.18 & 0.15 & 0.14 & 0.23 & 0.08 & 0.50 \\
\hline Apathy severity & $0.91^{*}$ & $<0.0001$ & 0.22 & 0.06 & 0.03 & 0.82 & 0.21 & 0.08 \\
\hline Disinhibition severity & $0.94^{*}$ & $<0.0001$ & $0.50^{*}$ & $<0.0001$ & -0.12 & 0.32 & 0.19 & 0.11 \\
\hline Irritability severity & $0.93^{*}$ & $<0.0001$ & $0.30^{*}$ & 0.01 & -0.11 & 0.36 & 0.07 & 0.54 \\
\hline Motor Disturbances severity & $0.99^{*}$ & $<0.0001$ & $0.28^{*}$ & 0.02 & 0.16 & 0.20 & $0.32^{*}$ & 0.01 \\
\hline Night time behavior severity & $0.94^{*}$ & $<0.0001$ & $0.38^{*}$ & 0.001 & $0.26^{*}$ & 0.03 & $0.30^{*}$ & 0.01 \\
\hline Appetite changes severity & $0.93^{*}$ & $<0.0001$ & -0.02 & 0.90 & 0.07 & 0.56 & 0.08 & 0.49 \\
\hline
\end{tabular}

$* \mathrm{p}>0.05$

$\dagger$ Spearman's correlation

Table 2: Association between BPSD severity and socio personal variables

\begin{tabular}{|l|c|c|c|c|c|c|c|c|c|c|}
\hline \multirow{2}{*}{ BPSD Severity } & \multicolumn{2}{|c|}{ Gender } & \multicolumn{2}{c|}{ Marital status } & \multicolumn{2}{c|}{ Type of family } & \multicolumn{2}{c|}{ Type of caregiver } & \multicolumn{2}{c|}{$\begin{array}{c}\text { Type of } \\
\text { accommodation }\end{array}$} \\
\cline { 2 - 13 } & $\mathbf{Z} *$ & $\mathbf{p}$ & $\mathbf{Z}$ & $\mathbf{p}$ & $\chi^{\mathbf{2}} \S$ & $\mathbf{p}$ & $\chi^{\mathbf{2}}$ & $\mathbf{p}$ & $\mathbf{Z}$ & $\mathbf{p}$ \\
\hline BPSD total & -1.31 & 0.19 & -1.54 & 0.12 & 2.70 & 0.26 & 0.13 & 0.94 & -1.94 & 0.05 \\
\hline Delusion severity & -0.36 & 0.72 & -0.37 & 0.71 & 1.82 & 0.40 & 0.12 & 0.94 & $-2.83^{*}$ & 0.01 \\
\hline Hallucination severity & $-2.07^{*}$ & 0.04 & $-1.93^{*}$ & 0.05 & 0.35 & 0.84 & $7.33^{*}$ & 0.03 & -0.12 & 0.91 \\
\hline Agitation severity & -0.87 & 0.39 & -0.53 & 0.60 & $6.40^{*}$ & 0.04 & $10.96^{*}$ & 0.004 & -0.09 & 0.93 \\
\hline Depression severity & -1.71 & 0.09 & -0.38 & 0.70 & 2.57 & 0.28 & 1.84 & 0.40 & -1.23 & 0.22 \\
\hline
\end{tabular}




\begin{tabular}{|l|c|c|c|c|c|c|c|c|c|c|}
\hline \multirow{2}{*}{ BPSD Severity } & \multicolumn{2}{|c|}{ Gender } & \multicolumn{2}{|c|}{ Marital status } & \multicolumn{2}{c|}{ Type of family } & \multicolumn{2}{c|}{ Type of caregiver } & \multicolumn{2}{c|}{$\begin{array}{c}\text { Type of } \\
\text { accommodation }\end{array}$} \\
\cline { 2 - 12 } & $\mathbf{Z}+$ & $\mathbf{p}$ & $\mathbf{Z}$ & $\mathbf{p}$ & $\chi^{2} \S$ & $\mathbf{p}$ & $\chi^{2}$ & $\mathbf{p}$ & $\mathbf{Z}$ & $\mathbf{p}$ \\
\hline Anxiety severity & -0.92 & 0.36 & -0.98 & 0.33 & 4.91 & 0.09 & 1.02 & 0.60 & -0.38 & 0.70 \\
\hline Euphoria severity & -0.97 & 0.33 & $-2.01^{*}$ & 0.04 & 0.46 & 0.80 & 1.42 & 0.49 & -0.48 & 0.63 \\
\hline Apathy severity & -0.99 & 0.32 & -1.18 & 0.24 & 1.34 & 0.51 & 0.76 & 0.69 & -1.11 & 0.27 \\
\hline Disinhibition severity & -0.38 & 0.71 & -0.78 & 0.43 & $8.09^{*}$ & 0.02 & 2.11 & 0.35 & -0.79 & 0.43 \\
\hline Irritability severity & -0.64 & 0.52 & -0.68 & 0.50 & 3.51 & 0.17 & 4.28 & 0.12 & $-2.25^{*}$ & 0.03 \\
\hline $\begin{array}{l}\text { Motor Disturbances } \\
\text { severity }\end{array}$ & $-1.99 *$ & 0.05 & $-3.06^{*}$ & 0.002 & 0.22 & 0.90 & 2.43 & 0.30 & -0.96 & 0.34 \\
\hline $\begin{array}{l}\text { Night time behavior } \\
\text { severity }\end{array}$ & -1.269 & 0.21 & -1.79 & 0.07 & 2.52 & 0.28 & 0.41 & 0.82 & -1.48 & 0.14 \\
\hline Appetite changes severity & -1.622 & 0.11 & -0.61 & 0.54 & 2.67 & 0.26 & 4.63 & 0.10 & -0.18 & 0.86 \\
\hline
\end{tabular}

${ }^{*} \mathrm{p}>0.05$

Mann Whitney U § - Kruskal Wallis

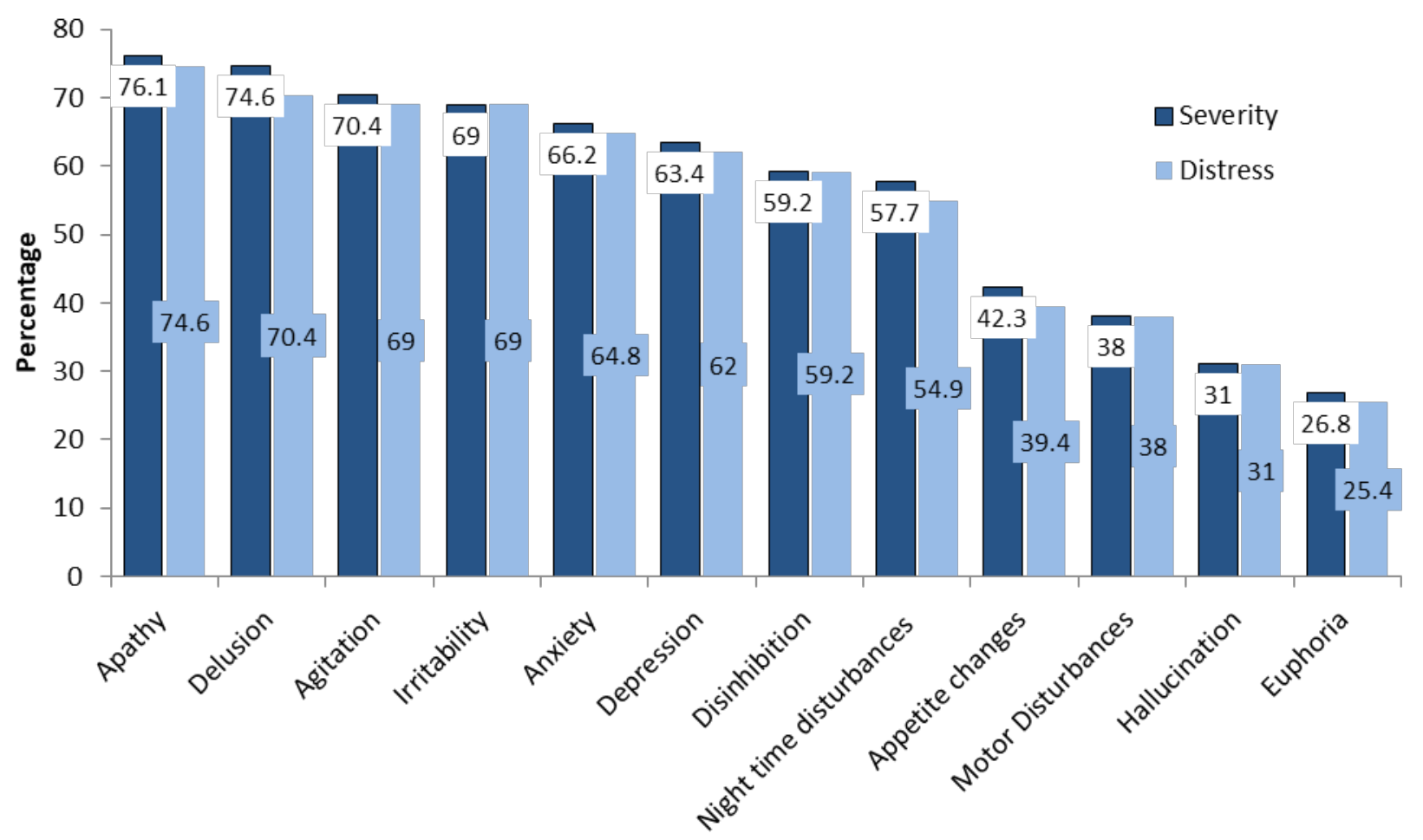

Fig 1: Distribution of BPSD severity and distress among patients with dementia 


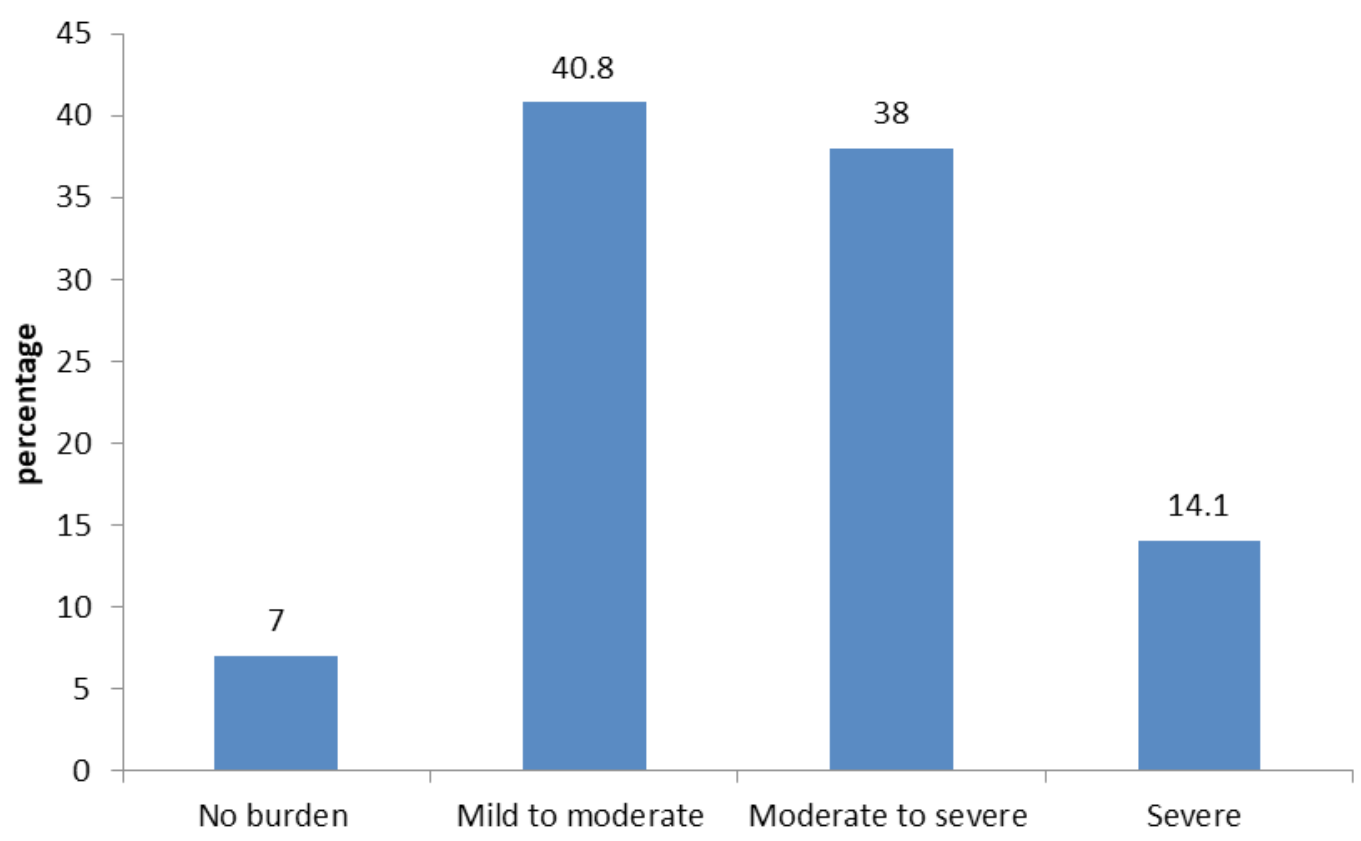

Fig 2: Burden among caregivers of patients with dementia

\section{Discussion}

This study is one among the few studies which examined the pattern of BPSD and caregiver burden among dementia patients in south India. It was ascertained that persistence of BPSD is associated with subsequent caregiver burden which needs further prospective exploration.

In our sample, mean age of patients was 80.03 years and $75 \%$ of them were females. Such percentages are similar to those reported in another study by Brunelle HL et al. ${ }^{16}$ Since the risk for dementia increases with age, most of the dementia studies reported a mean age of 75 to 85 years. As life expectancy of women is higher; most of the studies reported an increased percentage of females having dementia which is also confirmed by present study. Caregivers' demographic characteristics also were matching with study findings by Kim Het al. ${ }^{17}$ Being a developing country, India has a conventional family system where women in family as the prominent caregiver is being reflected in this finding. $35.2 \%$ of patients were fully dependent compared to $50 \%$ in a previous study which indicates our study population is more independent. ${ }^{18}$

The distribution of most common BPSD symptoms in a study by Baharudin $\mathrm{AD}$ et $\mathrm{al}^{19}$ such as apathy, irritability and agitation is fairy matching with the current study findings. Hsu $\mathrm{TJ}$ et $\mathrm{al}^{20}$ reported rarely found BPSD symptoms were mainly euphoria, motor disturbances, appetite changes and hallucinations which is also confirmed by the current study findings. There are findings that motor disturbances is also a common BPSD but the current study didn't support this. However, this study's finding confirms the universality of pattern in BPSD across the populations. This study also gives the understanding that apathy and agitation are the most common problem the family has to deal with. Current study reported a mean score of 42.25 in ZBI unlike studies which reported a mean score below 30 , which points out that burden is found to be high in current study population which could be also because of social factors where caregivers are responsible for household works as well as rearing of children in addition to caregiving. ${ }^{19}$

Current study confirmed that BPSD severity and distress are strongly correlated with caregiver burden similar to the reports of previous studies. Current findings can be interpreted that as age progresses, hallucination and night time behaviours tend to increase. Sensory deprivation and disturbed sleep due to ageing may add to the symptoms. But there are a few studies which contradicts the present study findings. Our findings also revealed that, like previous studies, delusion, hallucination, motor disturbances, and night time behaviour severity increases as dementia progresses ${ }^{21}$. Thus caregivers shall be educated that they should expect BPSD to be increased as dementia advances. There 
was a difference in hallucination, euphoria and motor disturbances severity between married and widowed patients. This could be a result of loss of partner, delayed grief, bereavement and loneliness in widowhood. There was a difference between nuclear family and joint family in agitation and disinhibition severity. This could be because of decreased perceived support and increased loneliness experienced by patients and caregivers in nuclear families. It was found that delusion and irritability severity was high in patients living at children's home. This could be due to difficulty in retaining new memories of changed home environment and anxiety of being in an unknown place. In the current study, hallucination was found to be higher in patients looked after by daughterin laws and higher agitation in patients looked after by spouses. This is in contrary to the findings of a previous study which said that no association was found between BPSD and type of caregivers. We can assume that less familiar caregivers like daughter in- law- may be a reason for hallucination and perceived freedom towards spouses might have increased the agitation.

Caregiver burden was found to be increased with duration of dementia. It adds to the current knowledge that burden increases with duration of caregiving. It can be explained in view of physical and mental exhaustion along with decreased support and increased financial burden for the caregiver as time progresses. Burden was found to be high in caregivers of fully dependent patients. It is expected as in addition to dementia care, caregivers were also supposed to do the palliative care of the patients which further increase the burden. No relationship was found with other sociopersonal variables of patients or caregivers.

\section{Conclusion}

The present study from Kerala shows that BPSD and caregiver burden is common among patients with dementia living in community. BPSD severity, distress and caregiver burden are strongly correlated to each other.

This study findings emphasise the need of screening of elderly for BPSD at community level. It also suggests the training of community health workers for identifying BPSD and also to teach patients and family how to manage them at home setting and also to support caregivers to reduce the burden. Improvising existing primary health care to elderly friendly especially to dementia clients is needed. Moreover, more research needs to be conducted on effect of pharmacological and non-pharmacological measures on BPSD and caregiver burden.

This study used purposive sampling which might have resulted in over or under representation of the symptoms. Another limitation is that the report of clinical diagnosis of dementia was lacking in many patients which we tried to minimize by cross checking the treatment documents.

This study helped to understand the magnitude of BPSD and caregiver burden among dementia patients living in community and their associated factors. It will help to plan and implement appropriate strategies to manage BPSD and caregiver burden in patients through training of community health workers and forming health care policies.

\section{Conflicting Interest (If present, give more details): Nil}

\section{Source(s) of Funding: Self}

Ethical Clearance: The research proposal was submitted to the institutional ethics committee and obtained permission. (IEC NO.B1/312/2015/ CONTSR(2) Dated 15.07.2015).

\section{References}

1. Lutz W, Sanderson W, Scherbov S. The coming acceleration of global population ageing.Nature. 2008;451:716-19.

2. Mental health of older adults; Fact sheet. World Health Organization. 2017.

3. World Health Organization. Global action plan on the public health response to dementia 2017-2025. 2017.

4. Prince M, Wimo A, Guerchet M, Ali G, Wu Y, Prina M. World Alzheimer Report. The global impact of dementia. An analysis of prevalence, incidence, cost \& trends. London: Alzheimer's Disease International (ADI). 2015.

5. Nichols E, EI Szoeke C, Vollset SE, Abbasi N, Abd-Allah F, Abdela J, Eddine Aichour MT et al. Global, regional, and national burden of Alzheimer's disease and other dementias, 19902016: a systematic analysis for the Global Burden of Disease Study 2016. The Lancet Neurology. 2018;18(1):88-106. 
6. Shaji KS, Jotheeswaran AT, Girish N, Srikala Bharath, Amit Dias, Meera Pattabiraman, Mathew Varghese. (Eds) The Dementia India Report: prevalence, impact, costs and services for Dementia: Executive Summary. Alzheimer's \& Related Disorders Society of India. 2010. New Delhi.

7. Tewari S. Ageing India: South India Oldest, Northeast Youngest. India Spend. 2016.

8. Haupt M, Kurz A, Janner M. A 2-year followup of behavioral and psychological symptoms in Alzheimer's disease. Dementia and Geriatric Cognitive Disorders. 2000;11(3):147-52.

9. Cooper C, Livingston G. Mental health/psychiatric issues in elder abuse and neglect. Clin Geriatr Med. 2014;30(4):839-50.

10. Hall KS, Hendrie HH, Brittain $\mathrm{HM}$ et al. The development of a dementiascreeing interview in two distinct languages. Int JMeth Psychiatric Res. 1993;3: 1-28.

11. Prince M, Acosta D, Ferri CP, Guerra M, Huang Y, Jacob KS, Rodriguez JJL et al. A brief Dementia screener suitable for use by non-specialists in resource poor settings; the cross-cultural derivation and validation of the brief Community Screening Instrument for Dementia. Int J Geriatr Psychiatry. 2011;26:899-07.

12. Cummings JL, Kaufer DI, Ketchel P, Smith V, MacMillan A, Shelley T, Lopez OL, DeKosky ST. Validation of the NPI-Q, a brief clinical form of the Neuropsychiatric Inventory. J Neuropsychiatry Clin Neurosci. 2000;12(2):233-39.

13. Wong A, Cheng ST, Lo, Eugene SK, Kwan, PWL, Law LSN, et al. Validity and Reliability of the Neuropsychiatric Inventory Questionnaire Version in Patients with Stroke or Transient Ischemic Attack Having Cognitive Impairment. Journal of Geriatric Psychiatry and Neurology. 2014;27(4):247-52.
14. Zarit, SH, Reever, KE, Bach-Peterson J. Relatives of the impaired elderly: Correlates of feelings of burden. The Gerontologist. 1980;20(6):649-655.

15. Seng BK, Luo N, Ng WY, Lim J, Chionh HL, Goh J, Yap P. Validity and reliability of the Zarit Burden Interview in assessing caregiving burden. 2010;39(10):758-63. Ann Acad Med Singapore

16. Brunelle-Hamanna L, Thiviergea S, Simarda M. Impact of a cognitive rehabilitation intervention on neuropsychiatric symptoms in mild to moderate Alzheimer's disease. Neuropsychological Rehabilitation. 2014.

17. Kim H, Chang M, Rose K, Kim S. predictors of caregiver burden in caregivers of individuals with dementia. Journal of advanced nursing. 2012; 68(4):846-55.

18. Wang J, Xiao LD, Li X, Bellis AD, Ullah S. Caregiver distress and associated factors in dementia care in the community setting in China. Geriatric Nursing. 2015;36(5):348-54.

19. Baharudin AD, Din NC, Subramaniam P, Razali R. The associations between behavioral psychological symptoms of dementia (BPSD) and coping strategy, burden of care and personality style among lowincome caregivers of patients with dementia. BMC Public Health. 2019;19(4):447-49

20. Hsu TJ, Tsai HT, Hwang AC, Chen LY, Chen LK. Predictors of non-pharmacological intervention effect on cognitive function and behavioral and psychological symptoms of older people with dementia. Geriatr Gerontol Int. 2017;17(1): 28-35.

21. Spalletta G, Musicco M, Padovani A, Rozzini L, Perri R, Fadda L et al. Neuropsychiatric symptoms and syndromes in a large cohort of newly diagnosed, untreated patients with Alzheimer disease. American Journal of Geriatric Psychiatry. 2010;18(11):1026-35. 